\title{
Analyse des conditions de rupture des canalisations des réseaux maillés due au phénomène de coup de bélier
}

\author{
Jawhar Gargouri ${ }^{1}$, Ezzeddine Hadj-tä̈eb ${ }^{1, a}$, Christian Schmitto ${ }^{2}$ et Guy Pluvinage ${ }^{2}$ \\ 1 UMF, École Nationale d'Ingénieurs de Sfax, BP 1173, 3038 Sfax, Tunisie \\ 2 LaBPS, École Nationale d'Ingénieurs de Metz, Technopole, Route d'Ars-Laquenexy, 57078 Metz Cedex 3, France
}

Reçu le 30 juin 2010, accepté le 7 mars 2011

\begin{abstract}
Résumé - Un modèle numérique, permettant d'obtenir de manière automatique des informations sur la propagation des ondes de coup de bélier dans les réseaux maillés de conduites, est présenté. Le modèle tient compte des bifurcations et des dérivations dans les réseaux de conduites ainsi que des pertes de charges. Il est constitué d'un système de deux équations aux dérivées partielles non-linéaires de type hyperbolique résolu par la méthode des caractéristiques. L'algorithme numérique ainsi construit fournit une estimation des pressions maximales dans le fluide et des contraintes maximales dans les parois dues à la fermeture rapide de vannes. Dans certains cas, la contrainte maximale peut devenir supérieure à la contrainte admissible et provoque la rupture des conduites. La dangerosité d'un défaut de type cratère de corrosion a été analysée en déterminant la distribution des contraintes en tête de ce défaut. Les résultats obtenus permettent de calculer le facteur d'intensité de contraintes d'entaille appliqué. Cette grandeur est insérée dans un diagramme Intégrité-Rupture de type SINTAP et les nœuds en situation critique sont déterminés. Il y a risque de rupture si le facteur de sécurité est inférieur à 2 . Les résultats de calcul montrent que presque tous les nœuds du réseau analysé sont situés en dehors du domaine d'intégrité.
\end{abstract}

Mots clés : Coup de bélier / méthode des caractéristiques / contraintes / facteur d'intensité / rupture de conduites

\begin{abstract}
Failure conditions analysis of looped network pipes due to water hammer phenomenon. A numerical model has been established in order to simulate the propagation of the waterhammer wave into a looped water networks. This model gives information on water hammer waves taking into account the structure of the water network and the lost of pressure. The numerical algorithm gives the value of the maximal fluid pressure induced by water hammer provoked by fast closing valves into network and then the maximal stresses into pipes. These maximal stresses can overcome the admissible stress and lead to failure risks. In this case, the severity of a defect like corrosion crater has been estimate by computing under water hammer pressure the stress distribution at defect tip. This allows to computes the applied notch stress intensity factor. This parameter is introduced into the SINTAP failure assessment diagram into order to determine nodes of the network in critical situation. Conventionally, it is admitted that the risk of failure exists if this safety factor is less than 2. The results indicate almost nodes of the studied network are outside of safe zone in the Failure Assessment Diagram.
\end{abstract}

Key words: Waterhammer / method of characteristics / stressas / intensity factor / pipe rupture

\section{Introduction}

Le problème du rendement de la distribution des eaux potables a, récemment, fait l'objet de nombreux travaux de recherche $[1,2]$. Il est vrai que l'on estime que $30 \%$ de l'eau distribuée est perdue par le fait de fuites de canalisations. Ces fuites sont dues à la rupture d'éléments

\footnotetext{
${ }^{a}$ Auteur pour correspondance : Ezed.Hadj@enis.rnu.tn
}

des circuits d'adduction. La rupture des canalisations, phénomène assez fréquent en milieu urbain, s'amorce sur un défaut, dû à la corrosion ou autre, sous l'effet des contraintes engendrées par les coups de bélier.

Les études réalisées sur le phénomène de coup de bélier dans les réseaux maillés de conduites sont assez rares [3,4]. Ce phénomène se produit suite à une fermeture brusque d'une vanne et provoque des ondes de pression qui se 
propagent le long des conduites pouvant causer des dégâts assez importants dans les canalisations et les composants hydrauliques. Il est donc nécessaire de bien dimensionner les conduites ou d'installer des systèmes de protection et de contrôle des variations brusques de pression [5].

Le dimensionnement et la protection des canalisations sont basés sur la mécanique des fluides, l'analyse limite et la mécanique des ruptures [6]. Ils ont fait l'objet de nombreux codes de calcul. Ces derniers présentent l'inconvénient dans le cas de rupture consécutive à des coups de bélier, de ne pas prendre en compte l'influence d'une vitesse de déformation élevée qui conduit à une modification de la loi de comportement du matériau et à une baisse de la ténacité de celui-ci. Ces deux éléments constituent un risque aggravant pour l'intégrité des canalisations.

L'objectif de cette étude consiste à définir le risque de rupture d'une conduite dans un réseau maillé présentant un défaut superficiel de type cratère de corrosion.

Pour cette raison, nous avons développé, sur cette question, un code de calcul des écoulements transitoires basé sur la théorie des caractéristiques avec prise en compte des pertes de charge. Ce code permet de calculer avec précision l'évolution des ondes de pression, en fonction du temps, tout le long des canalisations d'un réseau maillé.

Dans une première étape, on s'intéressera à analyser les variations brusques de pression dans les canalisations résultant du phénomène de coup de bélier provoqué par la fermeture rapide d'une vanne. Les résultats obtenus permettent de calculer le pic de pression engendré et corrélativement le pic de contrainte résultant.

Dans une deuxième étape, ce pic de contrainte sera introduit dans un critère de dimensionnement basé sur le diagramme intégrité-rupture avec la courbe de rupture donnée par le code SINTAP pour définir le niveau de risque en terme de coefficient de sécurité et de situation critique des nœuds du réseau.

\section{Formalisme mathématique du phénomène de coup de bélier}

Dans le cas d'un écoulement unidirectionnel dans une conduite cylindrique de diamètre $D$ et d'épaisseur $e$, constituée d'un matériau au comportement élastique linéaire obéissant à la loi de Hooke, les relations de base de calcul du phénomène de coup de bélier sont représentées par les équations de continuité et de mouvement qui s'écrivent [7] :

$$
\frac{\partial H}{\partial t}+\frac{C^{2}}{g S} \frac{\partial Q}{\partial x}=0 \quad \text { et } \quad \frac{\partial Q}{\partial t}+g S \frac{\partial H}{\partial x}+g S J=0
$$

Dans cette formule, $Q$ est le débit, $H$ est la charge définie par $H=z+p /(\rho g), p$ est la pression, $z$ est la cote au sol, $S$ est la section de la conduite, $J=\lambda Q|Q| /\left(2 D S^{2}\right)$ est la perte de charge linéaire par unité de longueur de conduite, $\lambda$ est le coefficient de perte de charge linéaire, $\rho$ est la masse volumique du fluide, $g$ est l'accélération de

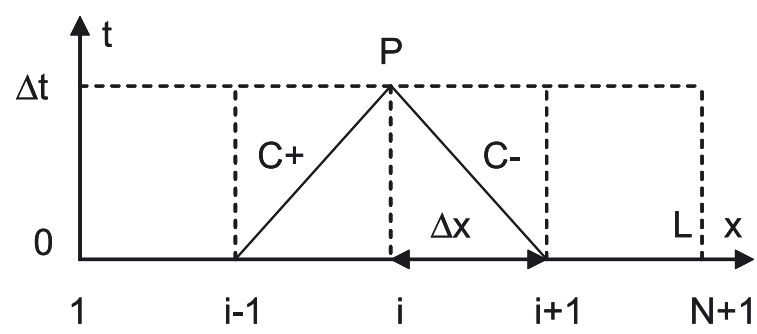

Fig. 1. Lignes caractéristiques : maillage régulier.

la pesanteur et $C$ est la célérité des ondes de pression qui peut s'exprimer par la relation :

$$
C=\left(\frac{\partial \rho}{\partial p}+\frac{\rho}{A} \frac{\partial A}{\partial p}\right)^{-1 / 2}=\left[\rho\left(\frac{1}{K}+c \frac{D}{e E}\right)\right]^{-1 / 2}
$$

où $K$ est le module de compressibilité du fluide, $E$ est le module de Young et $c$ est un paramètre caractérisant le type d'ancrage de la conduite et qui est fonction du coefficient de Poisson $\nu: c=\left(1-\nu^{2}\right)[8]$.

\section{Résolution numérique}

\subsection{Méthode des caractéristiques}

La méthode des caractéristiques [9] est appliquée pour transformer le système d'équations aux dérivées partielles (1) en un système d'équations différentielles ordinaires qui peuvent être intégrées numériquement sans difficulté. On obtient :

$$
\mathrm{d} Q \pm \frac{g S}{C} \mathrm{~d} H+g S J \mathrm{~d} t=0 \quad \text { et } \quad \mathrm{d} x= \pm C \mathrm{~d} t
$$

le signe + est attribué aux ondes provenant de l'amont et le signe - aux ondes provenant de l'aval.

Les équations (3) déterminent l'évolution du débit et de la charge en fonction du temps et de l'espace. Elles sont très appropriées pour être résolues numériquement sur micro-ordinateur. La solution ainsi obtenue constitue une solution du système original des équations (1).

L'intégration des équations (3), le long des lignes caractéristiques (Fig. 1), permet d'obtenir :

$$
\begin{aligned}
& Q_{\mathrm{P} i}-Q_{i-1}+\frac{g S i}{C_{i}}\left(H_{\mathrm{P} i}-H_{i-1}+J_{i-1} \Delta x\right)=0 \\
& Q_{\mathrm{P} i}-Q_{i+1}-\frac{g S i}{C_{i}}\left(H_{\mathrm{P} i}-H_{i+1}+J_{i+1} \Delta x\right)=0
\end{aligned}
$$

où $\Delta x$ est le pas d'espace du maillage régulier.

La pression est calculée par la formule :

$$
p_{P i}=\rho g\left(H_{\mathrm{P} i}-z_{i}\right)
$$

et la contrainte maximale est obtenue à partir de la pression maximale en un point de la paroi de la conduite à l'aide de l'expression :

$$
\sigma_{\max }=\frac{p_{\max } D}{2 e}
$$

où $e$ est l'épaisseur de la conduite au point de pression maximale $p_{\max }$ et $D$ son diamètre. 


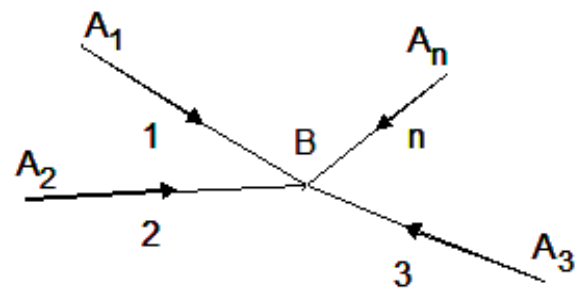

Fig. 2. Jonction de $n$ conduites.

\subsection{Calcul en un nœud avec branchement de plusieurs conduites}

Considérons donc un nœud $B$ auquel aboutissent $n$ tronçons d'extrémités $\mathrm{A}_{i}-\mathrm{B}$. Le débit dans chaque tronçon d'indice $i$ est désigné par $Q_{i}$. Le sens conventionnel de circulation des débits est choisi vers le nœud (Fig. 2)

En B, l'équation de continuité s'écrit donc :

$$
Q_{1 \mathrm{BP}}+Q_{2 \mathrm{BP}}+Q_{3 \mathrm{BP}}+\ldots Q_{n \mathrm{BP}}=0
$$

Sur chaque tronçon, on applique la théorie des caractéristiques. Pour une onde descendante aboutissant au nœud B, l'équation (4) donne :

$$
\frac{g S_{i}}{C_{i}} H_{\mathrm{BP}}+Q_{i \mathrm{BP}}=\frac{g S_{i}}{C_{i}} H_{\mathrm{A}_{i}}+Q_{i \mathrm{~A}_{i}}-\frac{g S_{i}}{C_{i}} j_{i}
$$

Notons que $j_{i}=J_{i} L_{i}$ est la valeur pondérée de la perte de charge le long du parcours de l'onde.

On peut écrire le système aux inconnues $H_{\mathrm{BP}}$ et $Q_{i \mathrm{BP}}$ sous la forme matricielle suivante :

$$
\begin{aligned}
& {\left[\begin{array}{ccccc}
1 & 1 & \ldots & 1 & 0 \\
1 & 0 & \ldots & 0 & \frac{g S_{1}}{C_{1}} \\
0 & 1 & \ldots & 0 & \frac{g S_{2}}{C_{2}} \\
\vdots & \vdots & \ldots & \ddots & \vdots \\
0 & 0 & \ldots & 1 & \frac{g S_{n}}{C_{n}}
\end{array}\right]\left\{\begin{array}{l}
Q_{1 \mathrm{~PB}} \\
Q_{2 \mathrm{~PB}} \\
\vdots \\
\vdots \\
\vdots \\
Q_{n \mathrm{~PB}} \\
H_{\mathrm{PB}}
\end{array}\right\}=} \\
& \left\{\begin{array}{l}
0 \\
\frac{g S_{1}}{C_{1}}\left(H_{\mathrm{A}_{1}}-j_{1}\right)+Q_{1 \mathrm{~A}_{1}} \\
\frac{g S_{2}}{C_{2}}\left(H_{\mathrm{A}_{2}}-j_{2}\right)+Q_{2 \mathrm{~A}_{2}} \\
\vdots \\
\frac{g S_{n}}{C_{n}}\left(H_{\mathrm{A}_{n}}-j_{n}\right)+Q_{n \mathrm{~A}_{n}}
\end{array}\right\}
\end{aligned}
$$

\section{Applications et résultats}

\subsection{Description du réseau maillé}

Afin de valider le modèle numérique proposé, nous examinons l'écoulement transitoire dans un réseau maillé

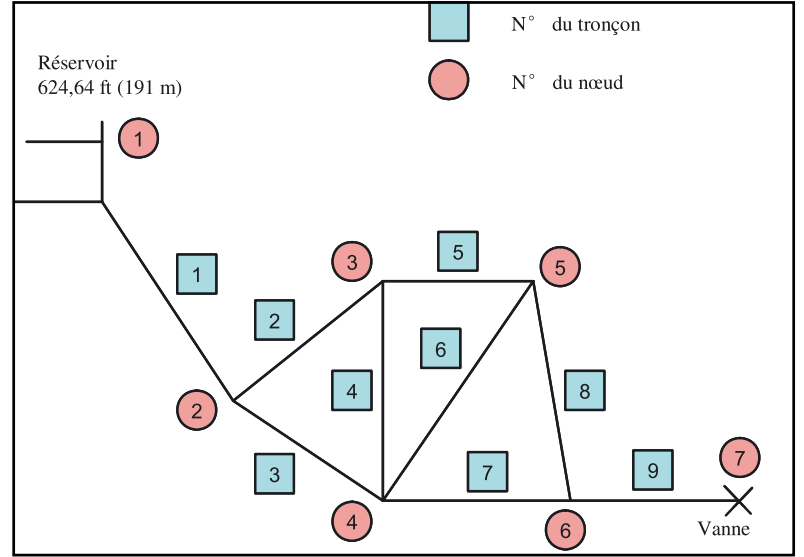

Fig. 3. Réseau maillé de conduites [3].

de conduites schématisé sur la figure 3 . Il s'agit d'un réseau à 3 mailles, 7 nouds et 9 tronçons. L'écoulement transitoire dans ce réseau a été étudié par [3]. Selon les valeurs des célérités qui varient de 914,4 à 1219,2 m.s ${ }^{-1}$, les conduites sont supposées élastiques, quasi-rigides et en fonte grise.

Bien que le réseau maillé choisi soit simple, le programme de calcul élaboré permet de simuler numériquement des réseaux maillés plus complexes, comportant des vannes, des réservoirs à niveau constant et des pompes. Les méthodes peuvent être appliquées à ces réseaux, bien que les pertes de charges seront plus importantes et amortissent très rapidement les fluctuations de pression.

Pour le réseau choisi, les pressions provoquées par le phénomène de coup de bélier sont très importantes comme le montreront les résultats par la suite. Ils permettent donc d'expliquer clairement la méthode d'analyse de la rupture des canalisations présentée ultérieurement.

Pour assurer la représentation du réseau dans le programme de calcul, on peut donner les caractéristiques géométriques et hydrauliques en régime permanent des tronçons (Tab. 1) et des nœuds (Tab. 2).

Le réseau doit être décrit à partir d'un nœud initial généralement un réservoir à niveau constant $H(1)$ qu'on numérotera 1. Pour chaque tronçon, on doit préciser son numéro, le numéro du nœud amont et le numéro du nœud aval.

\subsection{Analyse des résultats}

Les évolutions, en fonction du temps, de la charge aux différents nœuds du réseau sont présentées sur la planche 1.

L'écoulement transitoire est provoqué par la fermeture brusque de la vanne au noud 7 .

Ces résultats concordent parfaitement avec ceux obtenus par [3] et mettent en évidence la complexité du phénomène de coup de bélier dans les systèmes de conduites à caractéristiques multiples. Toutefois, la lecture des figures est aisée et permet de juger si les surpressions sont admissibles ou non. 

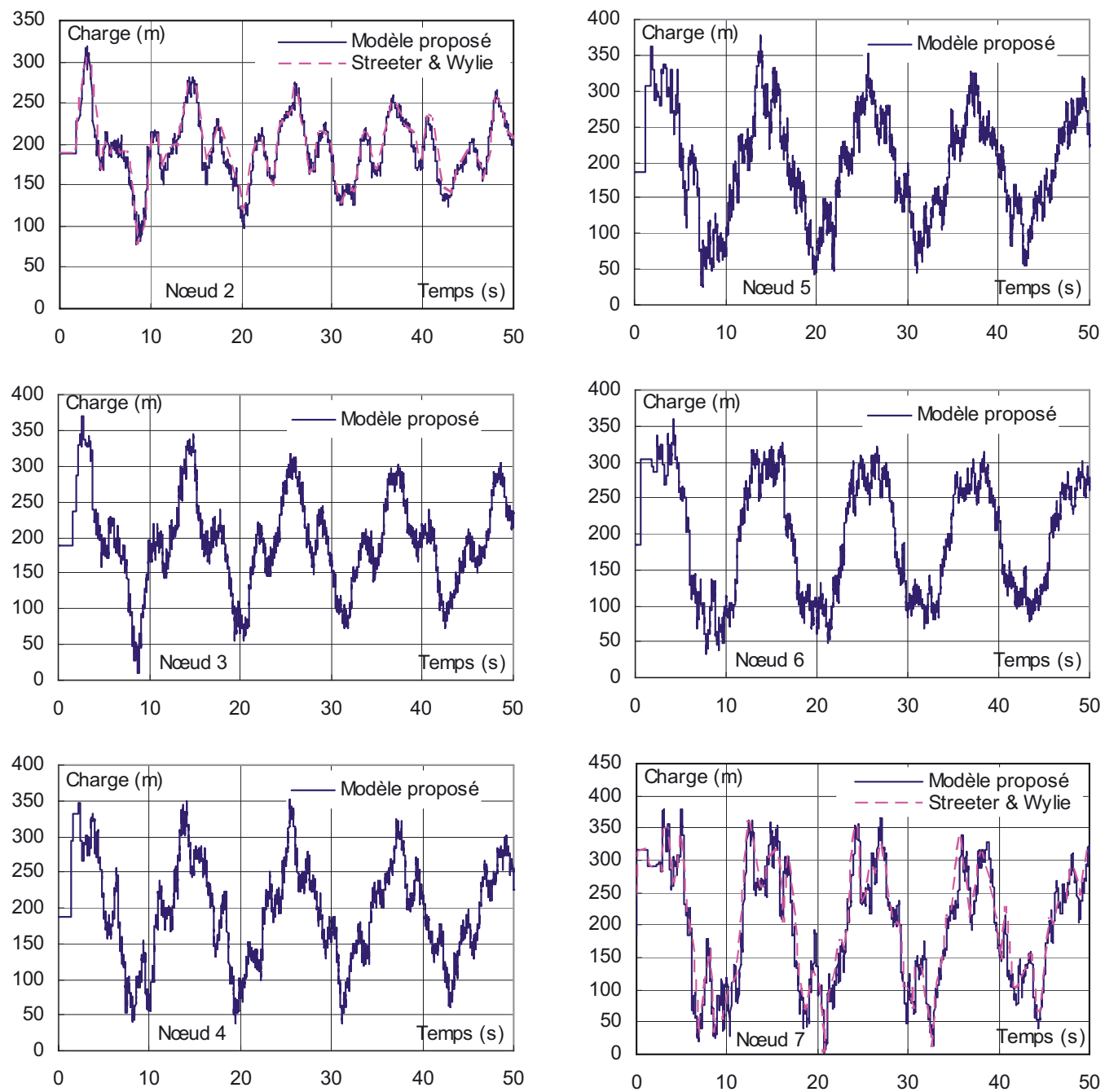

Planche 1. Évolution de la charge aux différents nœuds du réseau.

Tableau 1. Caractéristiques des tronçons.

\begin{tabular}{cccccc}
\hline NT & $L(\mathrm{~m})$ & $D(\mathrm{~mm})$ & $Q\left({\left.\mathrm{~L} . \mathrm{s}^{-1}\right)}^{\mathrm{C}\left(\mathrm{m} . \mathrm{s}^{-1}\right)}\right.$ & $\lambda$ \\
\hline 1 & 609,6 & 914,400 & 849,500 & 1005,8 & 0,03 \\
2 & 914,4 & 762,000 & 408,7016 & 1143,0 & 0,028 \\
3 & 609,6 & 609,600 & 440,7984 & 1219,2 & 0,024 \\
4 & 548,6 & 457,200 & 176,5611 & 914,4 & 0,02 \\
5 & 457,2 & 457,200 & 232,1405 & 1143,0 & 0,02 \\
6 & 487,7 & 457,200 & 111,1896 & 975,4 & 0,025 \\
7 & 670,6 & 762,000 & 506,1699 & 957,1 & 0,04 \\
8 & 457,2 & 609,600 & 343,3301 & 914,4 & 0,03 \\
9 & 609,6 & 914,400 & 849,500 & 1005,8 & 0,024 \\
\hline
\end{tabular}

Tableau 2. Caractéristiques des nœuds.

\begin{tabular}{ccc}
\hline Nœud & Altitude $z(\mathrm{~m})$ & Charge $H(\mathrm{~m})$ \\
\hline 1 & 191,000 & 191,0000 \\
2 & 0,000 & 189,2942 \\
3 & 0,000 & 187,9187 \\
4 & 0,000 & 186,5040 \\
5 & 0,000 & 185,8806 \\
6 & 0,000 & 184,2936 \\
7 & 0,000 & 182,9290 \\
\hline
\end{tabular}

Tableau 3. Contraintes maximales dues à une fermeture brusque au nœud 7 .

\begin{tabular}{ccccc}
\hline $\begin{array}{c}\text { Conduites en fonte } \\
\text { Diamètre } \\
(\mathrm{mm})\end{array}$ & $\begin{array}{c}\text { Épaisseur } \\
(\mathrm{mm})\end{array}$ & Nœud & $\begin{array}{c}P_{\max } \\
\text { (bar) }\end{array}$ & $\begin{array}{c}\text { Contrainte } \\
\text { maximale }(\mathrm{MPa})\end{array}$ \\
\hline 914,4 & 12,7 & 7 & 37,26 & 134,1 \\
914,4 & 12,7 & 6 & 35,33 & 127,2 \\
609,6 & 10,0 & 5 & 37,14 & 113,2 \\
762,0 & 11,4 & 4 & 34,53 & 115,4 \\
762,0 & 11,4 & 3 & 36,22 & 121,1 \\
914,4 & 12,7 & 2 & 32,59 & 117,3 \\
\hline
\end{tabular}

\subsection{Contraintes maximales}

Sur le tableau 3, on résume les valeurs numériques des pressions maximales et des contraintes maximales pour les différentes conduites du réseau. On constate que la contrainte maximale se produit au nœud 7 . Elle a pour valeur $134,1 \mathrm{MPa}$.

Pour une canalisation saine, c'est-à-dire sans défaut, le niveau de cette contrainte serait tout à fait acceptable 
Tableau 4. Propriétés mécaniques de la fonte grise.

\begin{tabular}{ccccc}
\hline $\begin{array}{c}\text { Limite d'élasti- } \\
\text { cité }(\mathrm{MPa})\end{array}$ & $\begin{array}{c}\text { Résistance } \\
\text { ultime }(\mathrm{MPa})\end{array}$ & $\begin{array}{c}\text { Allongement } \\
\%\end{array}$ & $\begin{array}{c}\text { Masse volumique } \\
\left(\mathrm{kg} \cdot \mathrm{m}^{-3}\right)\end{array}$ & $\begin{array}{c}\text { Ténacité } K_{\text {Ic }} \\
(\mathrm{MPa} \sqrt{ } \mathrm{m})\end{array}$ \\
\hline 300 & 420 & 10 & 7050 & 14,90 \\
\hline
\end{tabular}

puisqu'elle est plus de 2,2 fois inférieure à la limite d'élasticité du matériau, donnée dans le tableau 4. Un coup de bélier ne porterait pas atteinte dans ce cas à l'intégrité de la canalisation.

\section{Détermination du risque de rupture d'une canalisation du réseau}

Le risque de rupture d'une canalisation d'eau, présentant un défaut de type cratère de corrosion sous l'effet d'un coup de bélier peut être apprécié par la valeur du coefficient de sécurité. Cette rupture peut s'amorcer par la présence d'un défaut critique de taille $a_{\mathrm{c}}$ soumis à une contrainte critique $\sigma_{g, c}$ correspondant à la résistance de rupture du matériau de la conduite ou ténacité $K_{\text {Ic }}$ [10].

La condition de rupture peut s'écrire :

$$
f\left(a_{\mathrm{c}}, \sigma_{g, \mathrm{c}}, K_{\mathrm{Ic}}\right)=0
$$

Il est plus utile de transformer ce critère à trois paramètres en un critère à deux paramètres afin d'obtenir une représentation plane. D'où la condition :

$$
f\left(k_{r}, L_{r}\right)=0
$$

où $k_{r}$ est le facteur d'intensité de contraintes appliqué adimensionnel défini comme étant le rapport du facteur d'intensité de contraintes appliqué $K_{\text {app }}$ divisé par la ténacité $K_{\text {Ic }}$ :

$$
k_{r}=\frac{K_{\mathrm{app}}}{K_{\mathrm{Ic}}}
$$

La contrainte appliquée adimensionnelle $L_{r}$ est définie comme étant le quotient entre la contrainte maximale appliquée $\sigma_{g}$ et la contrainte d'écoulement (choisie soit comme la limite d'élasticité $\sigma_{Y}$, soit comme la résistance ultime $\sigma_{\mathrm{U}}$ ou encore comme la contrainte limite classique d'écoulement $\left.R_{c}=\left(\sigma_{\mathrm{U}}+\sigma_{Y}\right) / 2\right)$ :

$$
L_{r}=\frac{\sigma_{g}}{R_{\mathrm{c}}}
$$

Toute condition de chargement dans la structure, contenant un défaut, menant à une situation non critique, est décrite par point calculé dans le plan $\left(L_{r}, k_{r}\right)$.

Les coordonnées de ce point sont $\left(L_{r}^{*}, k_{r}^{*}\right)$. La rupture s'amorce ou se produit lorsque les coordonnées de ce point augmentent jusqu'à atteindre les valeurs critiques $\left(L_{\mathrm{rc}}, k_{\mathrm{rc}}\right)$.

Tous les points de fonctionnement critiques menant à la rupture sont situés sur une courbe de rupture $f\left(L_{r c}, k_{r c}\right)=0$ donnée par le code SINTAP niveau 1.

En d'autres termes, si le point de fonctionnement est à l'intérieur du Diagramme Intégrité Rupture qui est limité par la courbe de rupture, la structure est en sécurité.

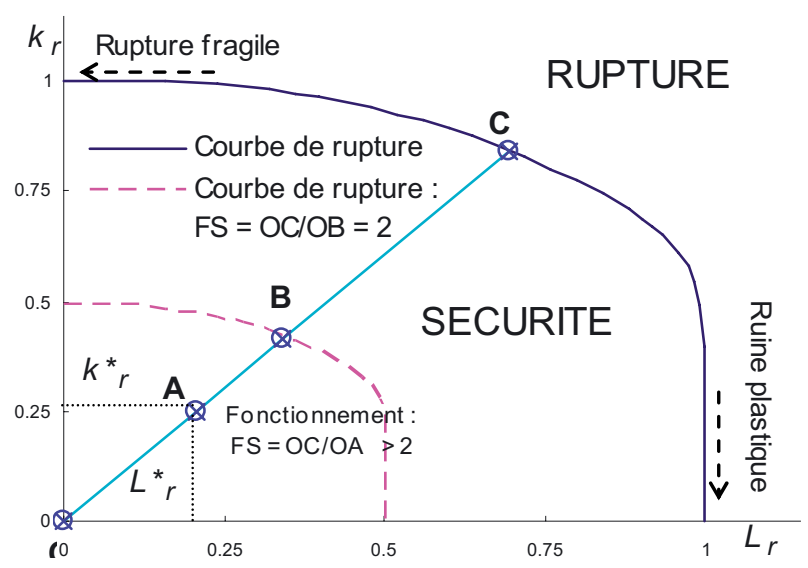

Fig. 4. Diagramme intégrité-rupture (DIR) typique indiquant la zone de rupture et le point de fonctionnement calculé.

Sinon, la rupture se produit car le point calculé se trouve à l'extérieur de la courbe de rupture (Fig. 4). Comme illustré sur cette figure, le Diagramme Intégrité Rupture permet de définir en outre le facteur de sécurité défini par le rapport OC sur OA pour un point de fonctionnement A.

Si le point de fonctionnement A est situé à l'intérieur de la zone délimitée par la courbe de rupture, le fonctionnement de la structure ne conduit à aucun risque de rupture.

Dans le passé, la courbe de rupture a été obtenue en supposant que toute rupture élastoplastique dérive de la rupture fragile par une correction plastique. Actuellement, la courbe de rupture s'obtient comme une limite inférieure de résultats expérimentaux et peut être légèrement différente selon les différents codes de rupture tels que EPRI (USA), R6 (UK), RCCMR (France) ou SINTAP (EU).

Pour la procédure SINTAP [11], qui sera utilisée dans ce qui suit, la courbe de rupture est donnée par l'équation suivante (pour le niveau 1) :

$$
\begin{gathered}
f\left(L_{r \mathrm{c}}\right)=\left[0,3+0,7 \exp \left(-0,6 L_{r \mathrm{c}}^{6}\right)\right] / \sqrt{\left[1+\frac{L_{r \mathrm{c}}^{2}}{2}\right]} \\
0 \leq L_{r \mathrm{c}} \leq L_{r \max } \\
L_{r \max }=1+\left[\frac{150}{\sigma_{Y}}\right]^{2,5}
\end{gathered}
$$

Dans cette étude les conduites considérées sont en fonte grise fragile ayant les propriétés mécaniques présentées dans le tableau 4.

Le défaut est supposé être une petite rayure de profondeur $a$ égale à l'épaisseur divisée par 200 et présentant un rapport d'aspect $a / c=0,4$. 
Tableau 5. Coordonnées des points de fonctionnement $\left(L_{r}^{*}, k_{r}^{*}\right)$.

\begin{tabular}{cccc}
\hline Nœud & $\begin{array}{c}\text { Épaisseur } \\
(\mathrm{mm})\end{array}$ & $\begin{array}{c}\text { Contrainte maximale } \\
(\mathrm{MPa})\end{array}$ & $\begin{array}{c}\text { Taille du } \\
\text { défaut }(\mathrm{mm})\end{array}$ \\
\hline 7 & 12,7 & 134,1 & 0,0635 \\
6 & 12,7 & 127,2 & 0,0635 \\
5 & 10 & 113,2 & 0,05 \\
4 & 11,4 & 115,4 & 0,057 \\
3 & 11,4 & 121,1 & 0,057 \\
2 & 12,7 & 117,3 & 0,0635 \\
\hline
\end{tabular}

\begin{tabular}{cccc}
\hline Noud & $K_{\text {app }}(\mathrm{MPa} \sqrt{ } \mathrm{m})$ & $k_{r}^{*}$ & $L_{r}^{*}$ \\
\hline 7 & 18,93 & 1,27 & 0,38 \\
6 & 17,96 & 1,20 & 0,36 \\
5 & 14,18 & 0,95 & 0,32 \\
4 & 15,43 & 1,03 & 0,32 \\
3 & 16,20 & 1,09 & 0,35 \\
2 & 16,56 & 1,15 & 0,36 \\
\hline
\end{tabular}

Cette hypothèse est très conservative. En général, les ruptures de canalisation sont des ruptures longitudinales. En prenant un rapport $a / c=0,4$ on se place dans un cas relativement défavorable.

Dans ces conditions, le facteur d'intensité de contraintes appliqué pour un tel défaut est donné par la relation :

$$
K_{\mathrm{app}}=\frac{M_{K} \sigma_{g} \sqrt{\pi a}}{\Phi_{d}}
$$

où $\Phi_{d}$ est l'intégrale elliptique de première espèce :

$$
\Phi_{d}=\sqrt{\left[1+1,464\left(\frac{a}{c}\right)\right]^{1,65}}
$$

$M_{K}$ est un facteur de correction géométrique exprimé par :

$$
M_{K}=\sqrt{\sec \left(\frac{\pi a}{B}\right)}
$$

La fonte grise est un matériau très fragile de sorte que tout défaut sera assimilé à une fissure elliptique.

Le facteur d'intensité de contraintes appliqué est calculé de l'équation (17) en considérant la contrainte maximale produite par le coup de bélier aux différents nœuds, ensuite les coordonnées du point de fonctionnement $\left(L_{r}^{*}, k_{r}^{*}\right)$ sont obtenues à partir des équations (13) et (14). Les valeurs calculées sont portées dans le tableau 5 et les points correspondants sont reportés dans le Diagramme Intégrité-Rupture de la figure 5, avec la courbe de rupture donnée par le code SINTAP niveau 1. De cette figure, nous constatons que tous les nouds sont en situation critique à l'exception du nœud 5 .

Ils sont situés, dans le domaine d'intégrité, au dessus de la courbe de rupture. Ceci est dû au matériau constitutif qui est très fragile. Dans plusieurs pays, la fonte grise est considérée comme matériau à « problèmes ».

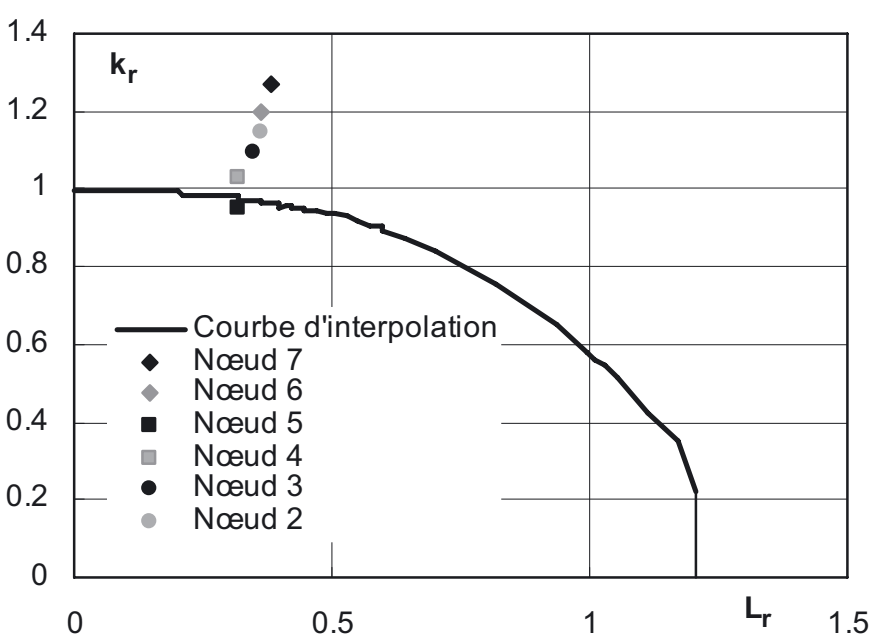

Fig. 5. Diagramme intégrité-rupture basé sur la procédure SINTAP et les points de fonctionnement correspondant aux nœuds de 2 à 7 .

En effet, à l'examen du tableau 5, il apparaît que c'est au nœud 5 que la contrainte est la moins élevée.

Les coordonnées du point de fonctionnement du noud 5 sont données dans le tableau 5 également.

En reportant ce point de fonctionnement dans le Diagramme Intégrité Rupture, il apparaît que celui-ci se trouve sous la courbe de rupture donnée par le code SINTAP, et donc dans le domaine d'intégrité et de coefficient de sécurité $\mathrm{OC} / \mathrm{OA}$ supérieur à 2 .

Une stratégie d'aménagement est souvent nécessaire pour remplacer toutes ces anciennes conduites.

Mais, une telle opération est généralement très coûteuse et sa réalisation demande souvent beaucoup de temps.

De toute façon, le vieillissement des conduites en fontes est dû essentiellement à l'action de la corrosion qui se manifeste par des piqûres de corrosion ou des cratères de corrosion (l'altération des propriétés mécaniques dans le temps est relativement faible).

Ces défauts de corrosion sont pris en compte dans la modélisation.

Il faut noter aussi que la corrosion de ces conduites peut se développer sous l'effet des contraintes engendrées par les coups de bélier et l'apparition du phénomène de cavitation de vapeur [12].

\section{Conclusion}

Cette étude nous a permis d'élaborer un modèle numérique pour calculer les contraintes maximales dans les réseaux maillés de conduites provoquées par le phénomène de coup de bélier.

La méthode présentée ci-dessus permet de suivre avec précision l'évolution des ondes de pression et en particulier d'apprécier l'amortissement de la surpression dans la propagation des ondes transmises lorsque l'écoulement porteur permanent est parfaitement défini. 
Basée sur des formules analytiques relativement simples issues de la théorie des caractéristiques, la méthode proposée permet de traiter, à l'aide d'ordinateurs, les écoulements transitoires dans les réseaux de conduites.

Elle permet directement d'examiner les conséquences de la réflexion d'ondes et de leur superposition. La méthode a été présentée en supposant que la perte de charge est une fonction quadratique du débit. Elle peut être utilisée avec toute autre hypothèse de dissipation d'énergie. À chaque hypothèse correspondrait un graphe universel permettant d'évaluer l'amortissement des ondes de pression dû à des manœuvres totales ou partielles.

Le calcul a permis de définir les circonstances les plus défavorables de fonctionnement du réseau en régime dynamique et d'en déduire les modifications nécessaires à apporter.

Un exemple d'application, concernant un réseau maillé, a été proposé. Il montre l'extension des possibilités de la méthode de calcul et notamment la possibilité de suivre systématiquement l'évolution des ondes de pression à travers les différentes bifurcations et les branchements du réseau.

La criticité d'un défaut dans les canalisations de type d'un cratère de corrosion supposé semi-elliptique et de profondeur très faible par rapport à l'épaisseur a été étudié à l'aide du Diagramme Intégrité Rupture avec la ligne d'interpolation donnée par le code SINTAP niveau 1. Nous constatons que tous les points sauf un sont situés en dehors du domaine d'intégrité. La procédure permet de conclure que l'ensemble du réseau étudié ne travaille pas dans le domaine de sécurité et il faut donc installer des systèmes de protection et de contrôle des variations brusques de pression.

\section{Références}

[1] M. Ferrante, B. Brunone, Pipe system diagnosis and leak detection by unsteady-state tests, 1 Harmonic analysis, Advances in Water Resources, Elsevier, 2003, Vol. 26, pp. $93-105$

[2] W. Mpesha, M.H. Chaudry, S.L. Gassman, Leak detection in pipes by frequency response method using a step excitation, J. Hydraul. Res. 40 (2000) 55-62

[3] V.L. Streeter, E.B. Wylie, Hydraulic transients, McGrawHill Book Compagny, New York, 1967

[4] H.M.V. Samani, A. Khayatzadeh, Transient flow in pipe networks, J. Hydraul. Res. 40 (2002) 637-644

[5] J. Gargouri, E. Hadj-Taïeb, C. Thirriot, Influence de l'élasticité de la paroi sur l'évolution des ondes de pression dans les réseaux de conduites, Mécanique \& Industries 9 (2008) 33-42

[6] G. Pluvinage, Mécanique Élastoplastique de la Rupture, Cepadues, 1989

[7] E.B. Wylie, V.L. Streeter, L. Suo, Fluid transients in system, Prentice Hall, New Jersey, 1993

[8] P. Ghilardi, A. Paoletti, Additionnal viscoelastic pipes as pressure surges suppressors, 5th International Conference on Pressure Surges, Paper E2, 1986, pp. 113-121

[9] J.A. Fox, Hydraulic Analysis of unsteady flow in pipe networks, The Mac Millan Press LTD., London, 1977

[10] G. Pluvinage, Fracture and fatigue emanating from stress concentrators, Éditeur Kluwer, 2001

[11] SINTAP Procedure, Final procedure: SINTAP (structural integrity assessment procedures) for European industry, Brite-Euram Project, BE95-1426, 1999

[12] L. Hadj Taïeb, E. Hadj Taïeb, Influence of non-linear elastic behaviour of pipes in transient flows with Vapour Cavitation, Mécanique \& Industries 9 (2010) 33-42 